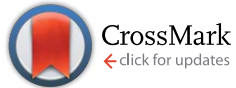

Cite this: Soft Matter, 2015, 11, 2268

Received 18th November 2014

Accepted 21st January 2015

DOI: $10.1039 / c 4 s m 02553 a$

www.rsc.org/softmatter

\section{Sphere to ring morphological transformation in drying nanofluid droplets in a contact-free environment}

\begin{abstract}
Ankur Miglani and Saptarshi Basu*
Understanding the transients of buckling in drying colloidal suspensions is pivotal for producing new functional microstructures with tunable morphologies. Here, we report first observations and elucidate the buckling instability induced morphological transition (sphere to ring structure) in an acoustically levitated, heated nanosuspension droplet using dynamic energy balance. Droplet deformation featuring the formation of symmetric cavities is initiated by capillary pressure that is two to three orders of magnitude greater than the acoustic radiation pressure, thus indicating that the standing pressure field has no influence on the buckling front kinetics. With an increase in heat flux, the growth rate of surface cavities and their post-buckled volume increase while the buckling time period reduces, thereby altering the buckling pathway and resulting in distinct precipitate structures. However, irrespective of the heating rate, the volumetric droplet deformation exhibits a linear time dependence and the droplet vaporization is observed to deviate from the classical $D^{2}$-law.
\end{abstract}

\section{Introduction}

Buckling of thin-walled structures is a ubiquitous phenomenon that is crucial to a plethora of natural processes and industrial applications. These range from drying of peas and corn kernels and the desiccation of pollen grains to invagination-controlled embryonic development (gastrulation process) in animals and the spray drying of suspensions for manufacturing pharmaceuticals, foodstuffs and functional coatings. ${ }^{1-6}$ In homogenous spherical shells subjected to uniform pressure, buckling instability initiates at a threshold pressure causing severe structural deformation in the form of surface cavities. The formation of these surface indentations leads to a significant reduction in the enclosed volume. However, imperfections in the shell (such as spatially varying thicknesses, geometrical topologies and material elastic properties) reduce the critical buckling pressure and time delay to buckling onset, thereby altering the buckling mode and the post-buckling shape. ${ }^{7-9}$ Understanding such modulated shell responses (commonly termed as imperfection sensitivity) is pivotal in preventing the catastrophic failure of monocoque engineering structures ranging from oil tanks and submarines to aircraft fuselages. ${ }^{10}$

Alternatively, the complex buckling patterns observed in drying suspensions are governed by a key precursor step involving surface agglomeration (structural skin formation)

Department of Mechanical Engineering, Indian Institute of Science, Bangalore, Karnataka-560012, India.E-mail: sbasu@mecheng.iisc.ernet.in and its subsequent deformation. ${ }^{\mathbf{1 1 - 1 3}}$ This involves a complex interplay of several thermo-physical processes such as surface regression, particle agglomeration and internal liquid phase circulation where the transfer of mass, momentum and energy are tightly coupled. By manipulating the timescales of these processes, either by varying functional properties (droplet size, initial solute concentration, particle size and dispersibility) or through external stimuli, the precipitation and buckling dynamics can be altered. ${ }^{14-16}$ This methodology has been exploited previously at different scales ranging from drying sessile droplets to manufacturing custom-engineered micro-capsules, lock and key colloids and shells with thickness at the nanoscale. ${ }^{17-19}$

In this study, however, we provide first insights into the buckling of nanoparticle (NP) laden droplets, drying in a contact-free environment (levitated using ultrasound). The buckling front kinetics observed here offer new insights compared to the previously reported data on the drying of levitated colloidal droplets ${ }^{\mathbf{2 0 - 2 3}}$ where no surface area reduction (or buckling process) was observed following a semi-solid crust formation (although solvent evaporation still proceeded through the porous shell). Through experiments and dynamic energy balance, we predict the rate of buckling (buckling front motion) and show how this varies with the heating rate leading to distinct ring micro-structures. The analysis is restricted only to externally heated droplets. However, for comparative evaluation, experimental measurements and physical estimates are presented for droplets vaporizing through the mechanism of acoustic streaming. 


\section{Materials and methods}

In order to study the buckling front kinetics, a single-axis ultrasonic levitator ( $100 \mathrm{kHz} ; 154 \mathrm{~dB}$ SPL) is used to suspend a $0.5 \pm 0.03 \mathrm{~mm}$ diameter (initial diameter $D_{\mathrm{i}}=2 R_{\mathrm{i}}$ ) nanofluid droplet (dispersed phase: nanosilica, base fluid: de-ionized water) at the centre antinode of a vertical standing wave. The droplet is irradiated with a tunable $(L=0-10 \mathrm{~W}), 10.6 \pm 0.03 \mu \mathrm{m}$ wavelength (infrared) $\mathrm{CO}_{2}$ diode laser (beam waist diameter of $3.5 \mathrm{~mm}$ ). Studies reported in the literature ${ }^{24}$ have shown that with directional radiative heating, the asymmetry in temperature profile is negligible for the laser flux magnitudes used in the current experiments.

The buckling instability driven morphological transition of the droplet featuring deformation of the consolidated shell is shown in Fig. 1-3. This process is captured using an ultra-high speed monochrome Photron SA5 camera coupled with a $5 \mathrm{X}$ Navitar zoom lens (temporal resolution $0.33 \mathrm{~ms}$; spatial resolution $\sim 0.6 \mu \mathrm{m}$ per pixel). The camera is synchronized with a laser using an external delay generator and placed orthogonal to

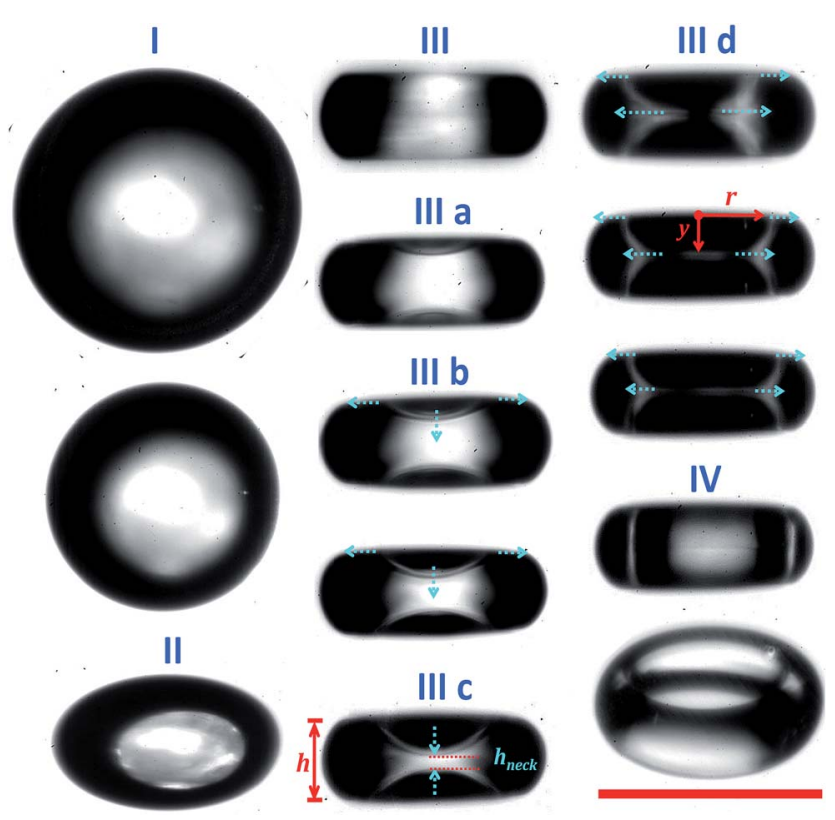

Fig. 1 Dynamic morphological transition during the vaporization and precipitation of an acoustically levitated laser irradiated nanosilica dispersion droplet at $I_{1}=0.104 \mathrm{MW} \mathrm{m}^{-2}$ : (I) uniform volumetric shrinkage, (II) onset of shape deformation (droplet squeezing) and subsequent transition from an oblate spheroid structure to (III) a disc with a toroidal edge; $t_{\mathrm{c}}=0 \mathrm{~s}$, (III a) onset of buckling; inversion of curvature with a characteristic degree of deformation $h^{*}=\frac{h_{\text {neck }}}{h} \approx 0.85 ; t_{\mathrm{c}}=0.176 \mathrm{~s}$, (III b) cavity growth with spreading in both the radial and axial directions: formation of the dimpled-disc structure $\left(0.08 \lesssim h^{*} \lesssim 0.85\right) ; t_{c}=0.276 \mathrm{~s}, 0.343 \mathrm{~s}$ and $0.51 \mathrm{~s}$, (III c) cavity growth purely in the longitudinal direction with constant radius at the rim, (III d) arrestation of the buckling fronts $\left(h^{*}=0\right)$ with subsequent cavity spread purely in the radial direction; $t_{\mathrm{c}}=0.593 \mathrm{~s}$, $0.77 \mathrm{~s}$ and $0.82 \mathrm{~s}(\mathrm{IV})$ merging of cavities and ring formation; $t_{\mathrm{c}}=0.87 \mathrm{~s}$. The blue arrows indicate the direction of depression growth. The scale bar equals $330 \mu \mathrm{m}$. (a)

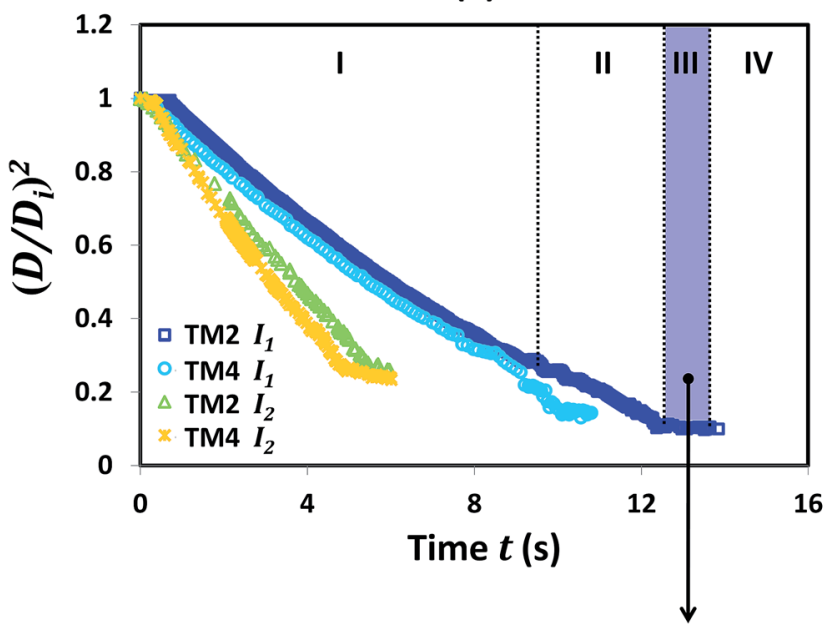

(b)

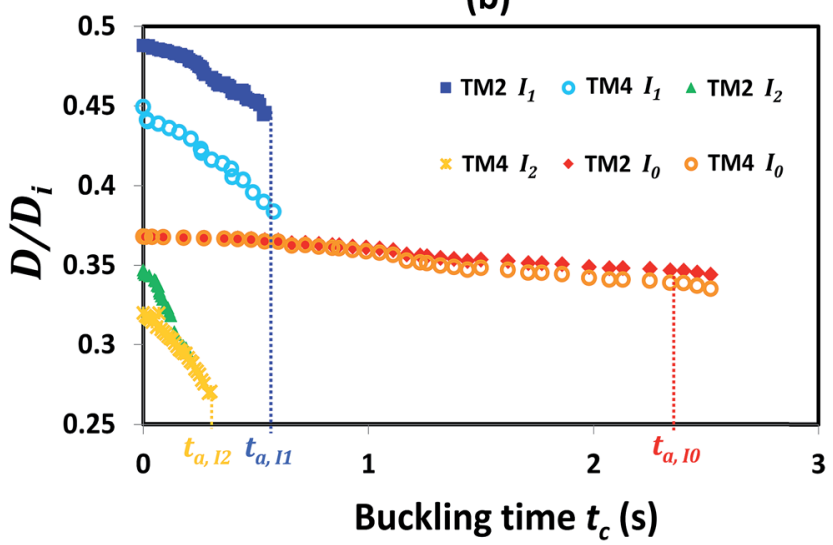

Fig. 2 (a) Time history of the squared non-dimensional droplet diameter $\left(D / D_{\mathrm{i}}\right)^{2}$ showing the distinct stages of the droplet lifecycle. (b) Temporal variation in the droplet size reduction during the buckling regime (III).

the laser beam axis (in the same horizontal plane). In this study, a stabilized, diluted dispersion of aqueous Ludox TM-40 nanosilica (from Sigma-Aldrich) with an average particle diameter $d=24 \pm 2 \mathrm{~nm}$ and dispersity $D \sim 0.3$ is investigated at two particle loading rates $c_{1}=0.764 \mathrm{vol} \%(2 \mathrm{wt} \%)$ and $c_{2}=1.547$ vol\% (4 wt\%) (the buckling behavior is thus studied only for dilute concentrations). Henceforth these concentrations will be referred to as TM2 and TM4 respectively. In order to form a stable, long-term suspension with homogenously dispersed NPs, the nanosuspension is subjected to ultrasound induced cavitation (sonication) in a constant temperature ice bath for $\sim 30$ min. Following sonication the zeta potential of the colloidal suspension is measured to be $\sim-50 \pm 2 \mathrm{mV}$ (using a zetaPALS: Phase Analysis Light scattering analyzer from Brookhaven Instruments), thus indicating a stable dispersion. These droplets are irradiated at three laser intensities: $I_{0}=0 \mathrm{MW} \mathrm{m}^{-2}$ (no heating), $I_{1}=0.104 \mathrm{MW} \mathrm{m}^{-2}$ and $I_{2}=0.208 \mathrm{MW} \mathrm{m}^{-2}$. The experiments were conducted at ambient temperature $(300 \mathrm{~K})$ and at a relative humidity $\mathrm{RH} \sim 60 \%$ (measured using a TSP01 USB temperature and relative humidity probe from Thor labs). 

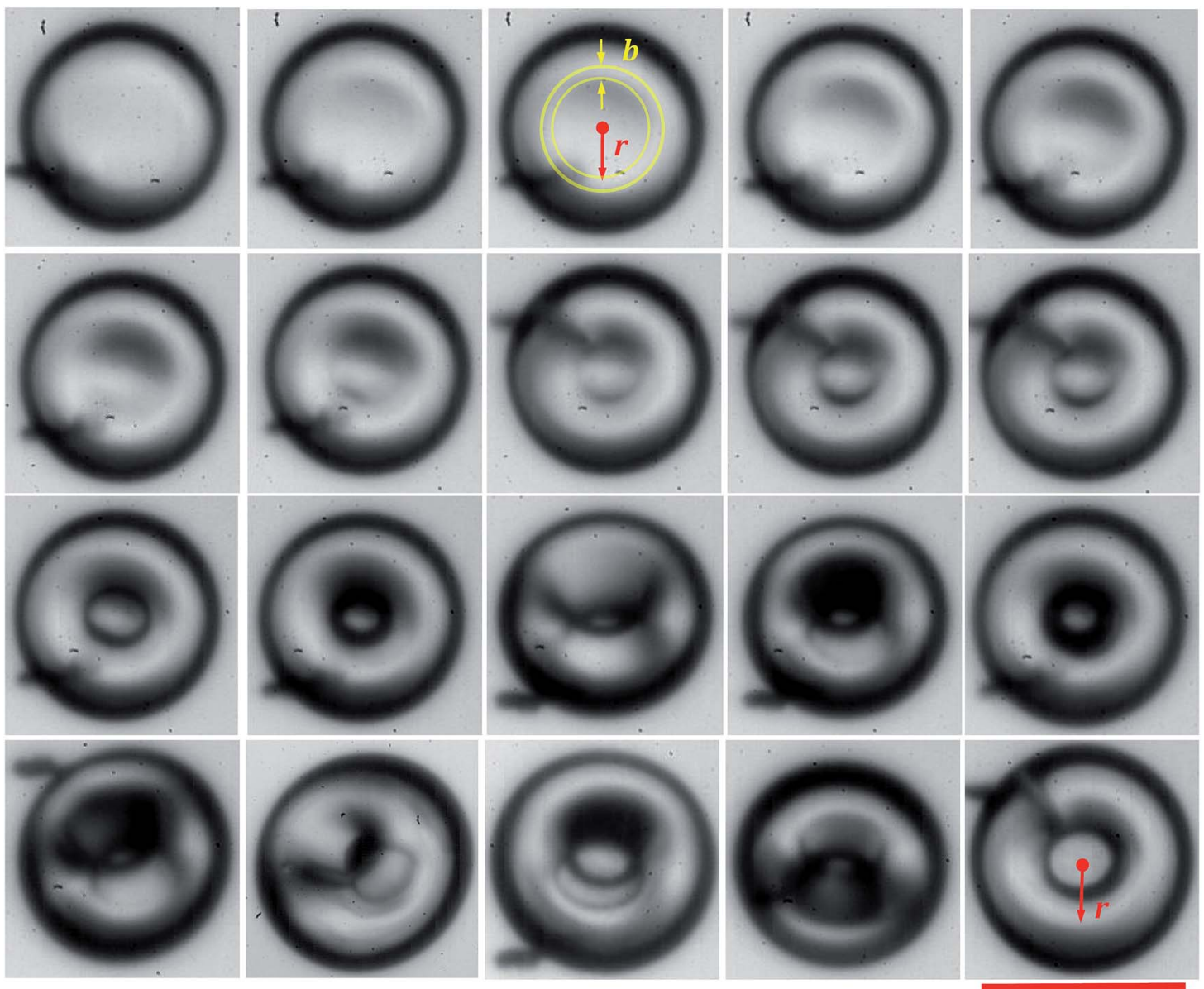

Fig. 3 High speed images showing the top view of the sequential cavity growth from buckling onset to doughnut-type final structure formation for acoustic streaming driven droplet vaporization $\left(I_{0}\right.$ : no heating). For the image sequence the snapshots are at times: $0,0.232,0.322,0.432$, $0.616,0.792,0.988,1.048,1.188,1.262,1.386,1.952,1.956,2.8,2.816,2.852,2.908,2.96,3.144$ and $3.296 \mathrm{~s}$. The scale bar equals $220 \mu \mathrm{m}$.

\section{Results and discussion}

\section{A Dynamic buckling process}

External heating initiates rapid solvent vaporization. The resulting solvent loss triggers a sequential three-stage droplet deformation process as shown in Fig. 1 and 2: (I) isotropic droplet shrinkage for the initial $65 \%$ of the total droplet lifetime $\left(t_{\mathrm{I}} \sim 0.65 t_{\mathrm{e}} ; t_{\mathrm{e}}\right.$ is the total evaporation time), (II) non-uniform Bernoulli pressure (acoustic radiation pressure) governed primary deformation phase. ${ }^{25-27}$ This process features droplet flattening and the gradual formation of an inhomogeneous porous shell with minimum thicknesses at the poles $\left(t_{\text {II }} \sim 0.25 t_{\mathrm{e}}\right)$. Finally, stage (III) corresponds to buckling of this shell. The mass diffusion Peclet number for the droplet is given by $\mathrm{Pe}_{\mathrm{m}}=\frac{t_{\text {Diffusion }}}{t_{\mathrm{e}}}=\frac{R_{\mathrm{i}}{ }^{2} / D_{\mathrm{a}}}{t_{\mathrm{e}}}$ and is in the range $5<\mathrm{Pe}_{\mathrm{m}}<250\left(D_{\mathrm{a}} \sim 5.6 \times 10^{-11} \mathrm{~m}^{2} \mathrm{~s}^{-1}\right.$ is the particle diffusion coefficient obtained from the Stokes-Einstein relation $\left.{ }^{28}\right)$. The high value of the Peclet number $\left(\mathrm{Pe}_{\mathrm{m}}\right)$ indicates that the timescale for homogenizing the internal composition (driven by particle diffusion) is one to two orders lower compared to the rate of droplet surface regression. This causes the dispersed nanoparticles to accumulate at the droplet sub-surface as the liquid-vapor interface recedes continuously, resulting in the formation of a viscoelastic shell. Inhomogeneity in the shell (spatially varying thickness) is however induced at two levels: (1) $\tau_{\mathrm{N}} \leq \tau_{\mathrm{S}}$ i.e. the average shell thickness $\tau_{\mathrm{N}}$ at the top (North Pole region $\mathrm{N}$ ) is nominally smaller than at the bottom $\tau_{\mathrm{S}}$ (South Pole region S). This differential thickness is induced by an acoustic streaming driven internal flow field which becomes asymmetric as the droplet shifts from the pressure node (due to the presence of the gravitational field). This asymmetric recirculation (with stronger recirculation in the bottom hemisphere) or orthokinetic particle transport mechanism causes preferential particle accumulation in the lower half and forms a thickened bottom structure. ${ }^{29-31}(2) \tau_{\mathrm{E}}>\tau_{\mathrm{N}}, \tau_{\mathrm{S}}$ i.e. the equatorial region $\mathrm{E}$ is thicker than the poles. Shell formation with non-uniform thickness is evident from the SEM micrographs (Fig. 4) of a representative case: a drying nanofluid droplet at dense NP concentrations $(c=10$ vol\%), under non lasing conditions. The cut section (along the meridional plane) through the final precipitate clearly illustrates heavy particle deposition along the equatorial belt while the shell narrows gradually at the top (near the North Pole) forming a weak circular sector that eventually buckles. The normalized average thickness $\tau_{\mathrm{E}} / R_{\mathrm{B}}$ is $\sim 0.26$ while in the bending strip region $\mathrm{b}$ 
(a)

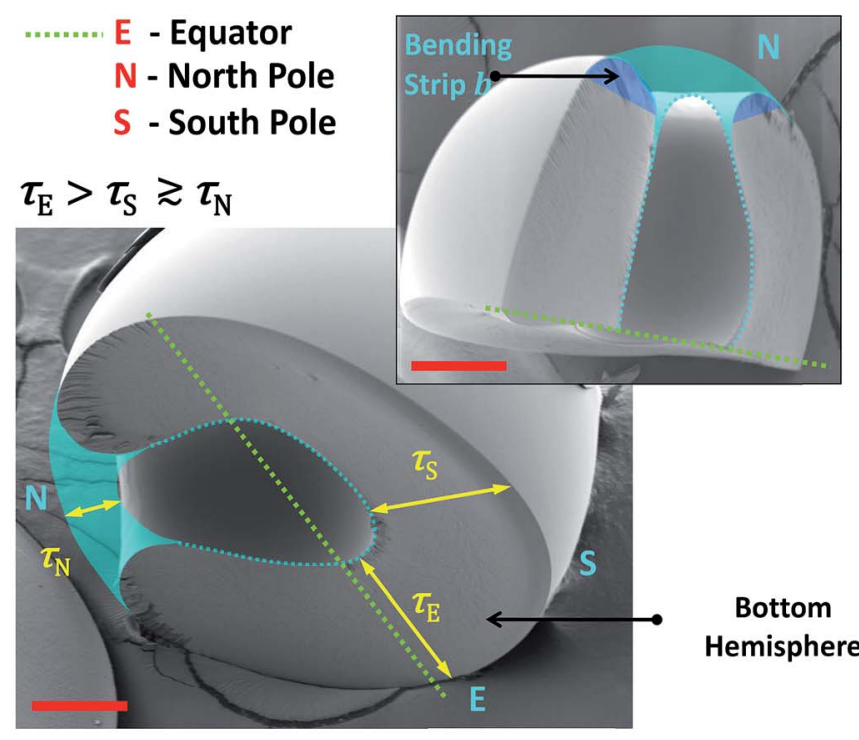

Inhomogeneous Shell structure

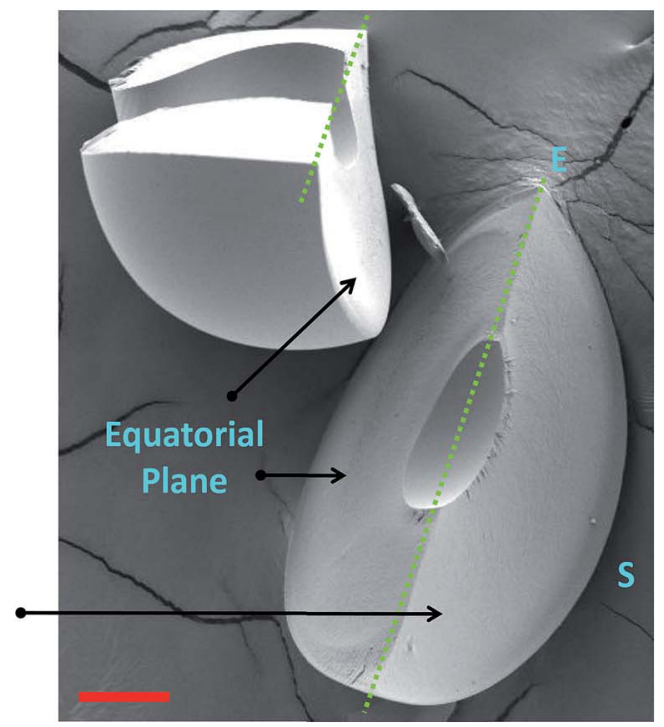

(b)

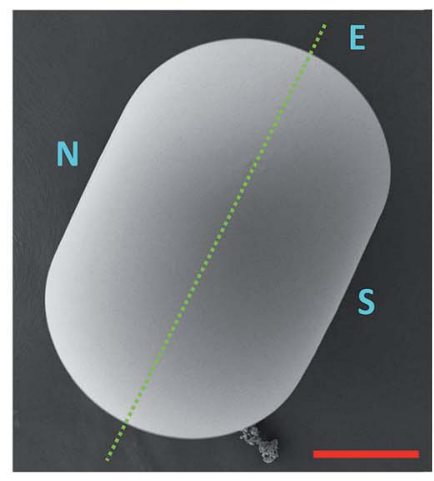

Weak soft spot in the polar region acting as a buckling site

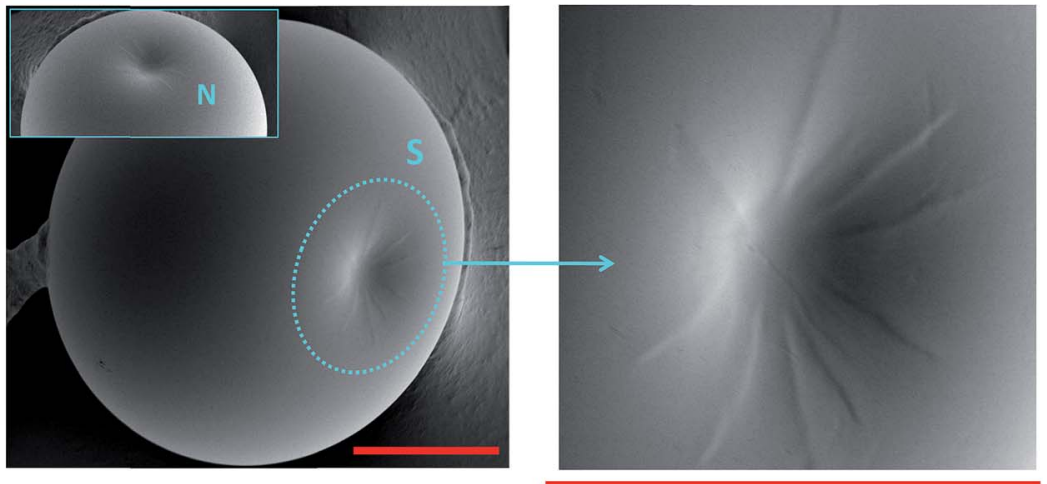

Fig. 4 (a) SEM micrographs showing a cut-section through the final precipitate microstructure of an acoustically levitated nanofluid droplet (with a dense particle loading rate of 10 vol\%) dried at non lasing conditions $\left(I_{0}=0 \mathrm{MW} \mathrm{m}^{-2}\right)$. (b) SEM images illustrating the precipitate structures obtained near buckling onset for a nanofluid droplet (TM2; $c_{1}=2 \mathrm{wt} \%$ ) dried at non lasing conditions $\left(I_{0}=0 \mathrm{MW} \mathrm{m}^{-2}\right)$. These indicate the locations of buckling sites (the weak, soft spots in the shell). All scale bars equal $50 \mu \mathrm{m}$.

surrounding the top collapsed region $\tau_{\mathrm{N}} / R_{\mathrm{B}} \sim 0.08$ and in the bottom sector $\tau_{\mathrm{S}} / R_{\mathrm{B}} \sim 0.18\left(R_{\mathrm{B}}\right.$ is the droplet radius at buckling onset). This indicates that the shell is $\sim 30-70 \%$ thinner near to the poles compared to the equator. Note that in the SEM images of Fig. 4(a), a classical gastrula structure (a term commonly used in embryology) is formed with only one sided (top) buckling. The caving in of the bottom portion is suppressed since at high NP loading rates $(10 \mathrm{vol} \%)$ the increased fluid viscosity dampens internal recirculation. This causes particle deposition in the bottom half through differential settling and leads to a thick, rigid bottom structure that resists buckling. However, at dilute concentrations $(c<2 \mathrm{vol} \%)$ considered in this study, both top and bottom buckling are observed (discussed subsequently) that form a ring. In summary, the shell thickness $\tau$ is a critical parameter that determines the stress required to initiate shell deformation and its inhomogeneity is induced such that the Polar regions become weak, soft spots and act as preferred buckling sites. The formation of these weak, soft spots near buckling onset is evident in Fig. 4b.

A first order estimate of the average shell thickness $\tau$ is obtained from particle mass conservation assuming that the concentration increases until a random packing fraction (vol\%) of $c_{\mathrm{f}}=0.56$ is achieved.

$$
\tau=R_{\mathrm{B}}\left[1-\left(\frac{c_{\mathrm{f}}-c_{\mathrm{i}}\left(R_{\mathrm{i}} / R_{\mathrm{B}}\right)^{3}}{c_{\mathrm{f}}-c_{\mathrm{i}}}\right)^{\frac{1}{3}}\right]
$$

$\tau$ obtained from eqn (1) is $\sim 15 \pm 7 \mu \mathrm{m}$ which is of the same order as that estimated from the SEM micrographs $(\sim 25 \mu \mathrm{m})$ of the final precipitates (Fig. 5). 

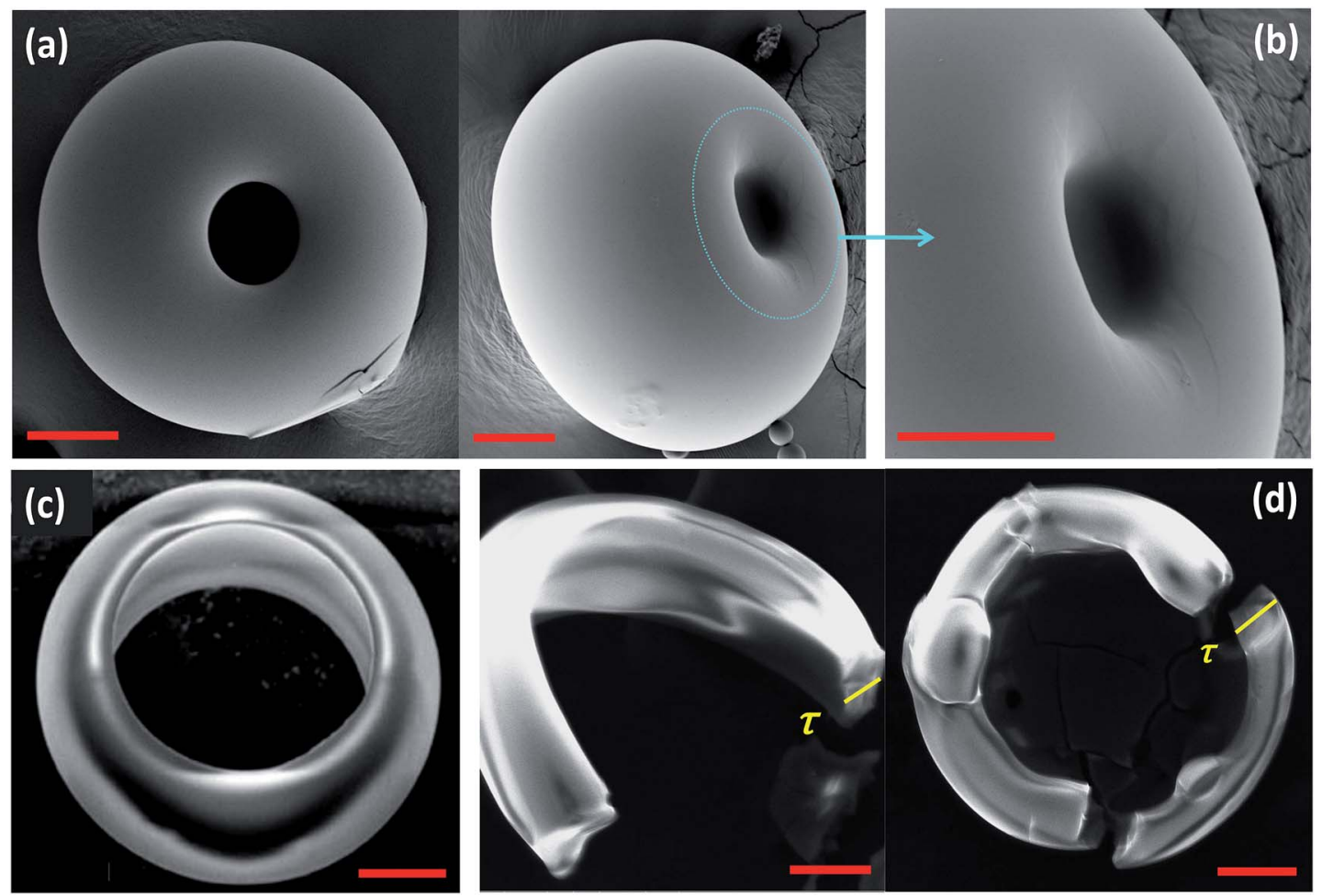

Fig. 5 SEM micrographs showing the final precipitate structures at three laser intensities: (a) no lasing; formation of a doughnut-type microstructure, (b) magnified view of the narrow bending strip region around the collapsed top sector; (c) $I_{1}=0.104 \mathrm{MW} \mathrm{m}^{-2}$; formation of a thick, smooth ring; and (d) $I_{2}=0.208 \mathrm{MW} \mathrm{m}^{-2}$; formation of thin, corrugated rings that fracture at higher mass diffusion Peclet numbers. All scale bars equal $50 \mu \mathrm{m}$.

Subsequently, in the secondary deformation phase (III) governed by meniscus or capillary forces, the thin-walled elastic shell with a Foppl-von Karman number $\alpha \approx 10\left(\frac{R_{\mathrm{B}}}{\tau}\right)^{2}(850 \leq \alpha$ $\lesssim 1150$ ) undergoes an inversion of the curvature at the poles marking buckling onset (III a). The resultant polar depressions grow and invaginate inside the droplet (Fig. 1) exhibiting a twosided, opposed, near-symmetric buckling (maximum difference in the time-averaged volumetric growth rate of the top and bottom cavities is $<10 \%)$. Particularly, the bottom depression growth is marginally slower due to its increased rigidity (as discussed previously). The time delay to buckling onset corresponds to a $\sim 93 \pm 4 \%$ reduction in the droplet volume and occurs during the final $1 \%$ and $5 \%$ of the droplet lifetime at $I_{0}$ and $I_{1}, I_{2}$ respectively.

In particular, the cavity growth during the secondary deformation proceeds in three stages (shown in Fig. 1 and later in Fig. 8d-f): (III b) in tandem radial-longitudinal expansion; in this regime, the radial spread rate of the cavity $(\dot{r})$ varies with the heating rate such that $\dot{r}_{I_{2}} \approx 2 \dot{r}_{I_{1}}>10 \dot{r}_{I_{0}}$; (III c) longitudinal expansion with a constant cavity-rim radius; and the final stage (III d) occurs following longitudinal cavity growth arrestation by the opposing buckling front; $\frac{h_{\text {neck }}}{h}=0$ (Fig. 1) with subsequent cavity tip flattening. This regime features a cavity expansion only along the radial direction, however, with different spreading rates at the rim plane (cavity top) and the equatorial plane (cavity bottom). The radial spread rate at the equatorial plane is significantly higher compared to that at the rim $(\sim 5$ times at $I_{1}$ and $\sim 2.5$ times at $I_{2}$ ). The end of stage III d corresponds to maximum shape deformation (the penultimate structure) prior to the merging of buckling fronts and the formation of the final ring-slot (IV).

At this point, the solvent has completely vaporized and the cavity shape is naturally stabilized by the opposing sister cavity, thereby controlling the ring-slot diameter $D_{\text {slot }}$ of the final precipitate structure (shown later in the inset of Fig. 7). The buckling fronts finally merge to form a ring microstructure $\left(t_{\mathrm{III}}\right.$ $\sim 0.1 t_{\mathrm{e}}$ ) as shown in the SEM images (Fig. 5). Without laser heating, however, regime III $d$ is absent and the transition from the cavity tip merging to the ring formation occurs in a single step. Thus, the occurrence of regime III substantiates the fact that the droplet surface regression continues even after porous shell formation, and the rate of surface retraction increases with the heating rate (as shown in Fig. 2b). Particularly, during the buckling phase (regime III), the time-mean droplet surface regression rate (characterized by the buckling front motion) $\frac{\mathrm{d} R}{\mathrm{~d} t}$ at $I_{2}$ is $O\left(10^{-2} \mathrm{~mm} \mathrm{~s}^{-1}\right)$. This is $\sim 3-4$ times faster compared to $I_{1}$ and an order of magnitude greater than at the non heating condition $I_{0}$ (where $R$ is the projected area equivalent radius).

\section{B Effect of heating rate on buckling front motion}

After buckling onset, the depression/cavity growth rate can be predicted from a global energy balance applied to a deformable control volume (CV) encapsulating the droplet (eqn (2) below). 
In particular, this analysis captures the temporal variation of the shell's bending deflection or motion of the cavity-tip $(y)$ and holds only until the cavity growth arrestation (i.e. the end of stage III c). Critical length scales involved in the analysis are shown in Fig. 6.

$$
\dot{Q}=2 \dot{W}_{\text {ext }}+\frac{\mathrm{d}\left(U_{\text {int }}\right)}{\mathrm{d} t}+2 \rho_{\mathrm{w}}\left(\frac{\mathrm{d} V_{\mathrm{c}}}{\mathrm{d} t}\right) h_{\mathrm{g}}
$$

where $\dot{Q}=I \pi R_{\mathrm{B}}^{* 2}$ is the external heat energy input effecting droplet gasification (where $R_{\mathrm{B}}^{*}$ is the projected area equivalent radius of the droplet and $I$ is the laser intensity). Factor 2 is added to the first and last terms due to the guided, symmetric folding of the top and bottom cavities. In eqn (2), convective heat transfer resulting from acoustic streaming (which also contributes to the droplet gasification) is neglected since it is negligible compared to the laser heat flux. This is indicated by significantly shorter (2-3 orders of magnitude) droplet evaporation time scales (i.e. total droplet lifetimes $\left.t_{\mathrm{e}}\right)$ at lasing conditions $\left(t_{\mathrm{e}} \sim 6 \mathrm{~s}\right.$ at $I_{2}$ and $t_{\mathrm{e}} \sim 15 \mathrm{~s}$ at $I_{1}$ ) compared to that under acoustic streaming driven vaporization $\left[t_{\mathrm{e}} \sim O(1000 \mathrm{~s})\right]$.

During the cavity growth phase (measured as the time period $t_{\mathrm{m}}$ from buckling onset to the merging of the top and bottom buckling fronts) the projected droplet aspect ratio $A R_{\mathrm{B}}=D_{0} / h$ (and hence the available projected area for heating) remains constant and the droplet exhibits no lateral contraction. Consequently, the change in structural morphology is solely due to the depression growth in the radial-longitudinal direction. Furthermore, as caving in proceeds, the amount of solvent vaporized at each instant corresponds to the volume displaced by the cavity. This solvent mass extracts latent heat and the resulting vaporization flux drains energy $\left(\rho_{\mathrm{w}}\left(\frac{\mathrm{d} V_{\mathrm{c}}}{\mathrm{d} t}\right) h_{\mathrm{g}}\right)$ from the droplet as it crosses the $\mathrm{CV}\left(h_{\mathrm{g}}\right.$ is the saturated vapor enthalpy at the spatially averaged droplet temperature $T_{\text {avg }}$ ). Thus, during the buckling transition, the micro-structure remains axisymmetric and the enclosed droplet volume reduces such that $2 V_{\mathrm{c}}+V_{\mathrm{d}}=$ constant $=V_{\mathrm{dB}}$ at each instant $\left(V_{\mathrm{dB}}\right.$ is the

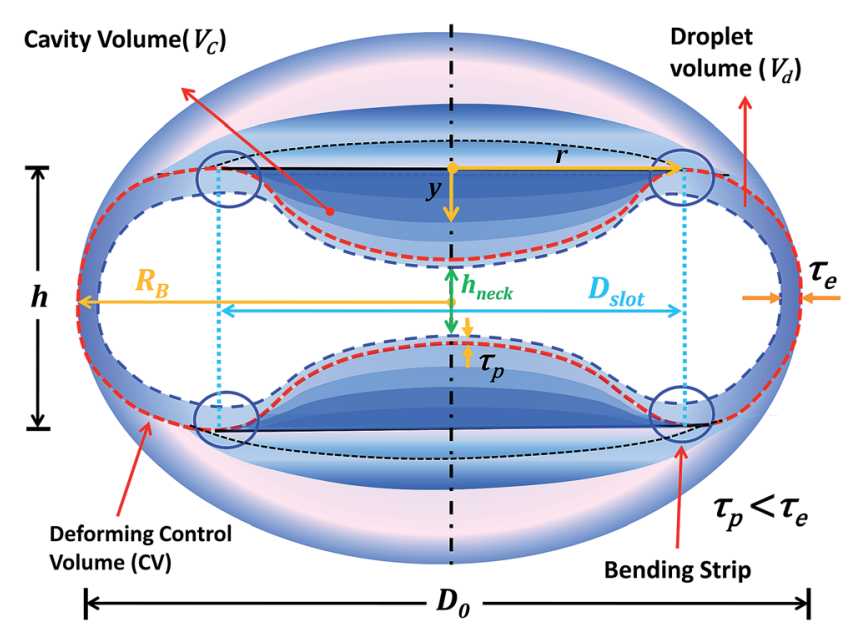

Fig. 6 Schematic showing the superimposed surface profiles of a buckling nanofluid droplet; critical buckling parameters are marked. volume of a flat, disc shaped structure with a toroidal edge that is formed at the buckling onset) or

$$
2 \frac{\mathrm{d} V_{\mathrm{c}}}{\mathrm{d} t}=-\frac{\mathrm{d} V_{\mathrm{d}}}{\mathrm{d} t}
$$

or cavity growth rate $=$ droplet deformation rate

The external moving boundary work is given by $\dot{W}_{\text {ext }}=-2\left(P_{\mathrm{B}}+P_{\mathrm{a}, \mathrm{rms}}\right) \frac{\mathrm{d} V_{\mathrm{c}}}{\mathrm{d} t}$, where $P_{\mathrm{B}}=\frac{\mu \mathrm{J} \tau}{k}$ is the capillary pressure drop (obtained from Darcy's law) that is required to percolate the solvent through a porous shell and drives the shell deformation. $J=\frac{\mathrm{d} R}{\mathrm{~d} t}$ is the solvent vaporization flux through the porous shell per unit surface area at buckling onset and is measured to be of the order of $10^{-6} \mathrm{~m} \mathrm{~s}^{-1}$ and $10^{-5} \mathrm{~m} \mathrm{~s}^{-1}$ at non lasing and lasing conditions, respectively. $k$ is the shell permeability obtained from the Kozeny-Carman equation $k=\frac{1}{180} \frac{\left(1-c_{\mathrm{f}}\right)^{3}}{c_{\mathrm{f}}{ }^{2}} d^{2}$ and $\mu$ is the dynamic viscosity of the continuous phase/solvent (water). It should be noted here that the solvent viscosity $\mu$ is considered in evaluating $P_{\mathrm{B}}$ since the fluid flowing through the pores would not carry any free NPs. Suspended NPs would deposit on the shell itself since the extremely small pore sizes would necessarily exclude their transport across the consolidated shell. However, the droplet viscosity increases continuously due to an increase in the surface concentration of the solute. This can be calculated as $\mu_{\mathrm{d}}=\mu\left(1+2.5 c_{\mathrm{B}}+6.5 c_{\mathrm{B}}{ }^{2}\right),{ }^{32}$ where $c_{\mathrm{B}}=c_{\mathrm{i}}\left(R_{\mathrm{i}} / R_{\mathrm{B}}\right)^{3}$ is the volumetric particle concentration at buckling onset.

At a critical magnitude of capillary pressure (or buckling pressure) the shell undergoes a sol-gel transition which marks the onset of buckling. At this point the deformation inducing, attractive capillary forces between nanoparticles (NPs) overcome the stabilizing electrostatic repulsive forces, thus transforming the shell from a viscous fluid to an elastic porous structure of densely packed particles. ${ }^{33}$ Based on Darcy's law, the order of magnitude of $P_{\mathrm{B}}$ is estimated to be $\sim 0.03 \mathrm{MPa}, 0.3$ MPa and $\sim 1 \mathrm{MPa}$ at the $I_{0}, I_{1}$ and $I_{2}$ laser settings, respectively. Thus, with an increase in the external heating rate the capillary pressure increases. At buckling onset $P_{\mathrm{B}}$ corresponds to an external critical buckling pressure given by the linear buckling shell theory i.e. $P_{\mathrm{cr}}^{*} \sim 10 \frac{Y}{\alpha}$ (where $Y$ is the shell's Young's modulus). $P_{\mathrm{cr}}^{*}$ is the threshold corresponding to the maximum of the shell's total free energy beyond which the cavity is in an unstable equilibrium state and grows spontaneously on its own accord, when $y \sim \tau .^{34}$ Indeed, our experimental measurements show that the inversion depth $y$ (corresponding to the line of maximum cavity deformation) is $\sim 7 \pm 1.5 \mu \mathrm{m}$ at $I_{0}, 9 \pm 2 \mu \mathrm{m}$ at $I_{1}$ and $8 \pm 2 \mu \mathrm{m}$ at $I_{2}$ which is of same order of magnitude as the shell thickness $\tau(\sim 15 \pm 7 \mu \mathrm{m})$ predicted from eqn (1). Given that the cavity radius $r \ll R_{\mathrm{B}}$ and based on the condition that the inversion depth $y \sim Y^{2} \tau^{5} / R_{\mathrm{B}}{ }^{4} P_{\mathrm{B}}{ }^{2}$ is of the same order as $\tau$ when the external pressure $P_{\mathrm{B}}$ equals the buckling pressure $P_{\mathrm{cr}}^{*}$, the Young's Modulus $Y$ is predicted to be in the range $\sim O(0.002-2$ $\mathrm{GPa}$ ). It will be shown later that even with a three orders of 
magnitude variation in $Y$ the prediction of the cavity growth rate remains unaltered.

Next, the RMS acoustic sound pressure of the standing wave is significantly smaller, $P_{\mathrm{a}, \mathrm{rms}}=0.02\left(10^{\mathrm{SPL} / 20}\right) \sim 1 \mathrm{kPa} \cdot{ }^{35} P_{\mathrm{B}} \gg P_{\mathrm{a}, \mathrm{rms}}$ indicates that $P_{\mathrm{B}}$ is the principle pressure force that triggers the buckling instability while the surrounding ultrasonic pressure field only plays a supporting role in the pre-buckling stages. Particularly, the acoustic field is responsible for droplet flattening and induces inhomogeneity in the shell. Shell deformation due to capillary forces arises principally due to the formation of several menisci with large interfacial curvatures (in the gaps between the particles). The resultant pulling tensile forces generated due to the curvature of these menisci pushes the particles inwards and pulls the solvent towards the outer periphery. ${ }^{33}$ As such, the buckling of the porous particle shell initiated by the capillary pressure appears to be a universal phenomenon in drying colloidal droplets. This is primarily governed by the shell formation process and exhibits a rather weak dependence on the environment since it has been previously observed in drying sessile droplets on a hydrophobic substrate, ${ }^{3}$ in levitated Leidenfrost droplets ${ }^{33}$ and in droplets sandwiched between circular glass slides. ${ }^{36}$

Note that since $P_{\mathrm{B}}$ is a direct measure of the severity of buckling and $\mathrm{Pe}_{\mathrm{m}}$ controls the shell formation process, the synergistic effect of these parameters determines the rate of cavity growth and the post-buckling reduction in droplet volume. In other words, these parameters govern the buckling pathway and the post-buckling final structure of the precipitate. This is indicated by a faster rate of increase of the relative cavity volume $\left(2 V_{\mathrm{c}} / V_{\mathrm{dB}}\right)$ with $P_{\mathrm{B}}$ and an inverse dependence of the buckling timescale $\left(t_{\mathrm{c}}\right)$ on $P_{\mathrm{B}}$ and $\mathrm{Pe}_{\mathrm{m}}$. These features are shown in Fig. 7 and indicate that higher heating rates result in thinner rings that tend to fracture due to higher $\mathrm{Pe}_{\mathrm{m}}$ (as shown in the SEM images in Fig. 5). The buckling timescale $t_{\mathrm{c}}$ at a high laser flux $I_{2}(\sim 0.2 \mathrm{~s})$ is $\sim 3$ times lower compared to that at $I_{1}(0.6 \mathrm{~s})$ and an order of magnitude lower than the case without heating ( $\sim 3 \mathrm{~s})$. Also, the experimentally measured time-averaged growth rate of the cavity tip is $(\dot{y})_{I_{0}} \approx 0.02 \pm 0.002 \mathrm{~mm} \mathrm{~s}^{-1},(\dot{y})_{I_{1}} \approx 0.11 \pm 0.018 \mathrm{~mm} \mathrm{~s}^{-1}$ and $(\dot{y})_{I_{2}} \approx 0.22 \pm 0.02 \mathrm{~mm} \mathrm{~s}^{-1}$. Since the cavity tip lies along the line of maximum deformation and $2 \dot{y}=-\dot{h}_{\text {neck }}$ (marked in Fig. 1), the magnitudes of $\dot{y}$ indicate that the rate of droplet necking doubles with laser intensity while it is an order of magnitude lower under pure vaporization. Similarly, the corresponding time-averaged, volume collapse rate $\left(\dot{V}_{\mathrm{d}}\right)$ at lasing conditions $\left(O\left(10^{-3} \mathrm{~mm}^{3} \mathrm{~s}^{-1}\right)\right)$ is an order of magnitude higher than at $I_{0}$. Also, the fraction of collapsed droplet volume at the point of arrestation $\left(t_{\mathrm{a}}\right)$ is $\sim 2$ times that under pure gasification. However, at the penultimate stage the percentage of buckled droplet volume at the high laser flux condition $\left(I_{2}\right)$ is $\sim 3$ times compared to that at $I_{0}$.

Next, the shell internal energy contribution comes from two components:

$$
U_{\text {int }}=m_{\mathrm{d}} c_{\mathrm{v}} T_{\mathrm{avg}}+2 E
$$

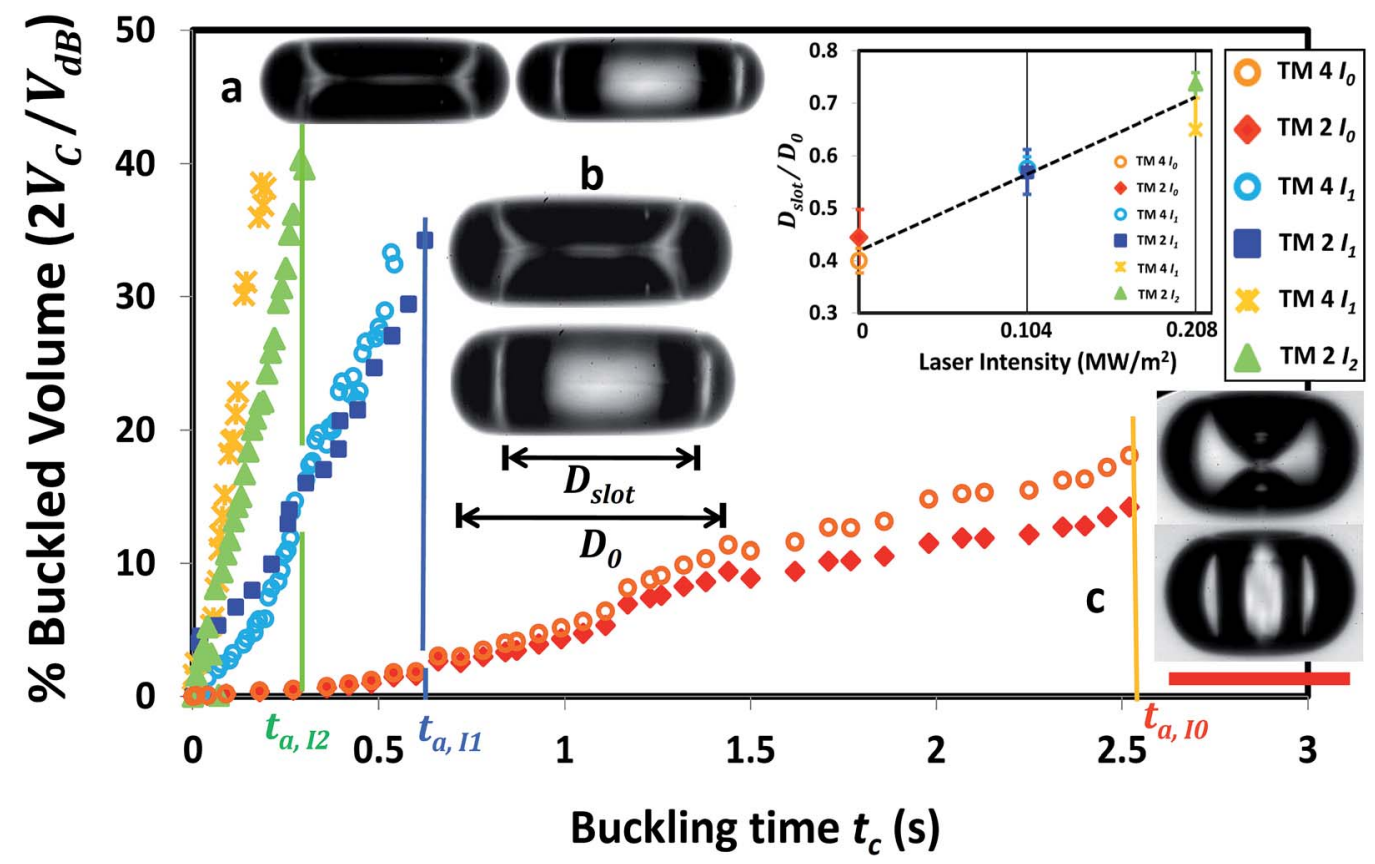

Fig. 7 Temporal variation of the normalized cavity volume $\left(2 V_{c} / V_{d B}\right)$ during the secondary deformation, buckling phase. Image pairs showing rapid transformation ( $\sim 1 \mathrm{~ms}$ ) from the penultimate structure to final ring slot formation; inverted plateau-type cavities with a high flatness ratio $f_{\mathrm{C}}=\frac{r-h / 2}{r}$ (a) $f_{\mathrm{c}} \sim 0.55$ at $I_{2}$ and $P_{\mathrm{B}} \sim 0.7 \mathrm{MPa}$, (b) $f_{\mathrm{c}} \sim 0.4$ at $I_{1}$ and $P_{\mathrm{B}} \sim 0.22 \mathrm{MPa}$; both resulting in broader ring slots, and (c) elongated, paraboloid wok-type cavities with $f_{\mathrm{c}} \sim 0.24$ at $I_{0}$ and $P_{\mathrm{B}} \sim 0.025 \mathrm{MPa}$ resulting in narrow slots. The inset shows the variation of the relative ring slot diameter $\left(D_{\text {slot }} / D_{0}\right)$ at these conditions. Buckling timescales are indicated by vertical lines and mark the point of cavity growth arrestation. The scale bar for all images equals $220 \mu \mathrm{m}$. 
where $m_{\mathrm{d}}(t)=\rho_{\mathrm{d}} V_{\mathrm{d}}$ is the time varying droplet mass and $\rho_{\mathrm{d}}$ is the droplet density. $E$ is the part of the internal energy associated with the morphology adopted by the shell. Particularly, it is the total elastic energy stored in the buckled shell that varies dynamically as the depth of the bulge increases continuously and the droplet attains different geometric variants of a dimpled-disc structure. It comprises four components:

$$
E=E_{0}+E_{\mathrm{BS}}+E_{\mathrm{I}}+E_{\mathrm{SS}}
$$

where, $E_{0}$ is the constant reference energy of the shell in its undeformed state, $E_{\mathrm{BS}}=E_{\mathrm{B}}+E_{\mathrm{S}} \approx 4 \pi r b Y\left(\frac{\tau^{2}}{R_{\mathrm{B}}{ }^{2}}\right) y$ is the total elastic energy stored in the narrow stress concentration rim (bending strip) of width $b$ and is linear in indentation depth or bending deflection $(y)$. This width, $b \equiv \frac{R_{\mathrm{B}}}{\alpha^{1 / 4}} \sim 32 \pm 10 \mu \mathrm{m}$, (shown in Fig. 3) physically represents an elastic length scale over which the deformations are localized and is obtained by the minimization of $E_{\mathrm{BS}}$ (the state corresponding to the equivalence of the bending and stretching energies: $\left.E_{\mathrm{B}}=E_{\mathrm{S}}\right)$. Here, $E_{\mathrm{B}} \sim 2 \pi r Y \varepsilon^{2}\left(\frac{\tau}{\alpha^{1 / 4}}\right) y$ and $E_{\mathrm{S}} \sim 2 \pi r Y\left(\frac{\tau}{\alpha^{3 / 4}}\right) y$ represent the energy contributions due to bending (out-of-plane) along the longitudinal direction and stretching (in-plane) along the circumferential directions respectively. During the inversion of curvature, the relative extension $\varepsilon=\frac{2 \theta R_{\mathrm{B}}-2 \theta\left(R_{\mathrm{B}}-\tau\right)}{2 \theta R_{\mathrm{B}}}=\frac{\tau}{R_{\mathrm{B}}}$ resulting from the compression of the outer layers and the stretching of the inner layers contributes to the elastic energy that is concentrated in the inverted cap (bulge) region $E_{\mathrm{I}} \sim Y\left(\varepsilon^{2}\right) \tau R_{\mathrm{B}} y \cdot{ }^{34}$ The last energy term $E_{\mathrm{SS}} \approx \frac{\pi}{16} Y \varepsilon y^{3}$ represents a contribution from the in-plane stretching (tensile stresses) that is generated along the cavity surface due to the cavity growth in the axial direction and represents the energetically favored buckling mode as the energy $E_{\mathrm{BS}}$ localized in the bending strip becomes enormous. This buckling pathway features preferential cavity growth in the longitudinal direction while the lateral width $(2 r)$ remains constant. ${ }^{37}$ During the cavity growth period $r>b$ (this is clearly seen in Fig. 3 in which $r \gtrsim 2.5 b$ ); this condition implies that $\frac{E_{\mathrm{BS}}}{E_{\mathrm{I}}} \approx \frac{r}{b}>1$ and $\frac{E_{\mathrm{SS}}}{E_{\mathrm{I}}} \approx\left(\frac{r}{b}\right)^{4} \gg 1$, thus, $E_{\mathrm{I}}$ has a negligible contribution in the total shell elastic energy. The internal energy change is then given by:

$$
\frac{\mathrm{d}\left(U_{\text {int }}\right)}{\mathrm{d} t}=m_{\mathrm{d}} c_{\mathrm{v}} \frac{\mathrm{d} T_{\text {avg }}}{\mathrm{d} t}+c_{\mathrm{v}} T_{\text {avg }} \frac{\mathrm{d} m_{\mathrm{d}}}{\mathrm{d} t}+2\left(\frac{\mathrm{d} E_{\mathrm{BS}}}{\mathrm{d} t}+\frac{\mathrm{d} E_{\mathrm{SS}}}{\mathrm{d} t}\right)
$$

It is noteworthy to mention here that buckling, as an instability, corresponds precisely to the lack of local energy balance between the pressure work, $P_{\mathrm{B}} \Delta V_{\mathrm{c}}$, and the shell elastic energy, $E$. The energy reduction in $P_{\mathrm{B}} \Delta V_{\mathrm{c}}$ dominates the energy cost of $E$ and as a result it is energetically favorable for the shell to collapse catastrophically and reduce its internal volume. Due to the short time scale of the cavity growth process $(<1 \mathrm{~s})$, the increase in the spatially averaged droplet temperature, $T_{\text {avg }}$, is nominally small $\left(<5{ }^{\circ} \mathrm{C}\right)$ and $T_{\text {avg }}$ at the laser flux conditions $I_{1}$ and $I_{2}$ is $\sim 40{ }^{\circ} \mathrm{C}$ and $\sim 52{ }^{\circ} \mathrm{C}$, respectively. Consequently, the sensible temperature rise has a negligible contribution to the internal energy change compared to the reduction resulting from mass transfer across the CV i.e. $m_{\mathrm{d}} c_{\mathrm{v}} \frac{\mathrm{d} T_{\text {avg }}}{\mathrm{d} t} \ll c_{\mathrm{v}} T_{\text {avg }} \frac{\mathrm{d} m_{\mathrm{d}}}{\mathrm{d} t}$ and the corresponding variation in the thermo-physical parameters $\left(\rho_{\mathrm{w}}=990 \pm 5 \mathrm{~kg} \mathrm{~m}^{-3}, c_{\mathrm{v}}=4.182 \mathrm{~kJ} \mathrm{~kg}^{-1} \mathrm{~K}\right.$ and $h_{\mathrm{g}}=$ $2580 \mathrm{~kJ} \mathrm{~kg}^{-1}$ ) due to the temperature change is less than $2 \%$. In addition, although the increase in the cavity radius occurs in three steps to achieve the final ring-slot radii (discussed in the previous section and shown in Fig. 8), as a first approximation we assume a time-averaged cavity radius $r^{*}=\frac{\int_{0}^{t_{a}} r \mathrm{~d} t}{t_{\mathrm{a}}}$ until buckling front arrestation and model the cavity shape with two potential surfaces of revolution of volume $V_{\mathrm{c}}=K r^{* 2} y$, (1) a paraboloid with $K=1.57$ and (2) a half-oblate spheroid with $K=$ 2.1. The time derivative of the internal energy given by eqn (6) reduces to:

$$
\begin{aligned}
\frac{\mathrm{d}\left(U_{\text {int }}\right)}{\mathrm{d} t}= & -\rho_{\mathrm{d}} c_{\mathrm{v}} T_{\text {avg }}\left(2 K r^{* 2}\right) \frac{\mathrm{d} y}{\mathrm{~d} t} \\
& +2\left(C_{0} 4 \pi r b Y \varepsilon^{2} \frac{\mathrm{d} y}{\mathrm{~d} t}+c_{2} \frac{3 \pi}{16} Y \varepsilon y^{2} \frac{\mathrm{d} y}{\mathrm{~d} t}\right)
\end{aligned}
$$

As the solvent vaporizes, the coupled mass loss causes a continuous change in the droplet density. Thus, $\rho_{\mathrm{d}}$ in eqn (7) is assumed to be a time-averaged density $\rho_{\mathrm{d}}=\left(\int_{0}^{t_{\mathrm{a}}} \rho \mathrm{d} t\right) / t_{\mathrm{a}}$ $\left(\rho_{\mathrm{d}}=1300 \pm 10 \mathrm{~kg} \mathrm{~m}^{-3}\right.$ and $1340 \pm 20 \mathrm{~kg} \mathrm{~m}^{-3}$ at $I_{1}$ and $I_{2}$ respectively). Under laser irradiation, the maximum changes in the shell elastic energy components given by $\frac{\mathrm{d} E_{\mathrm{BS}}}{\mathrm{d} t}$ and $\frac{\mathrm{d} E_{\mathrm{SS}}}{\mathrm{d} t}$ are $O\left(10^{-7}\right) \mathrm{J} \mathrm{s}^{-1}$ and $O\left(10^{-7}\right) \mathrm{J} \mathrm{s}^{-1}$ respectively at $I_{1}$ while the corresponding values at $I_{2}$ are $O\left(10^{-6}\right) \mathrm{J} \mathrm{s}^{-1}$ and $O\left(10^{-6}\right) \mathrm{J} \mathrm{s}^{-1}$ respectively. In contrast, the decrease in internal energy content due to the droplet mass reduction $-\rho_{\mathrm{d}} c_{\mathrm{v}} T_{\mathrm{arg}}\left(2 K r^{* 2}\right) \frac{\mathrm{d} y}{\mathrm{~d} t}$ is significantly larger, $O\left(10^{-2}\right) \mathrm{J} \mathrm{s}^{-1}$. Therefore, the net internal energy change is given by $\frac{\mathrm{d}\left(U_{\mathrm{int}}\right)}{\mathrm{d} t} \approx-\rho_{\mathrm{d}} c_{\mathrm{v}} T_{\text {avg }}\left(2 K r^{* 2}\right) \frac{\mathrm{d} y}{\mathrm{~d} t}$.

Similarly, as the solvent percolates and undergoes a phase change, the rate of energy transfer across the CV associated with solvent mass loss and thermal energy transported along with the vapor is four orders higher, $O\left(10^{-2}\right) \mathrm{J} \mathrm{s}^{-1}$. The rate at which displacement work is expended in deforming the droplet $-P_{\mathrm{C}} \frac{\mathrm{d} V_{\mathrm{d}}}{\mathrm{d} t}$ is $O\left(10^{-7}\right) \mathrm{J} \mathrm{s}^{-1}$ and $O\left(10^{-6}\right) \mathrm{J} \mathrm{s}^{-1}$ respectively at $I_{1}$ and $I_{2}$. Under pure vaporization, the deformation rate work is $\sim 3-4$ orders lower $\left(\right.$ i.e. $10^{-10} \mathrm{~J} \mathrm{~s}^{-1}$ ). These values are of the same order as the change in total shell elastic energy at their respective laser heat flux conditions. Therefore, the external work term is also negligible compared to the rate of change of internal energy $\frac{\mathrm{d}\left(U_{\text {int }}\right)}{\mathrm{d} t} \sim O\left(10^{-2}\right) \mathrm{J} \mathrm{s}^{-1}$. As such, as the droplet morphs to different deformed shapes the contribution to the overall energy budget either through work done by the capillary forces 
(a)

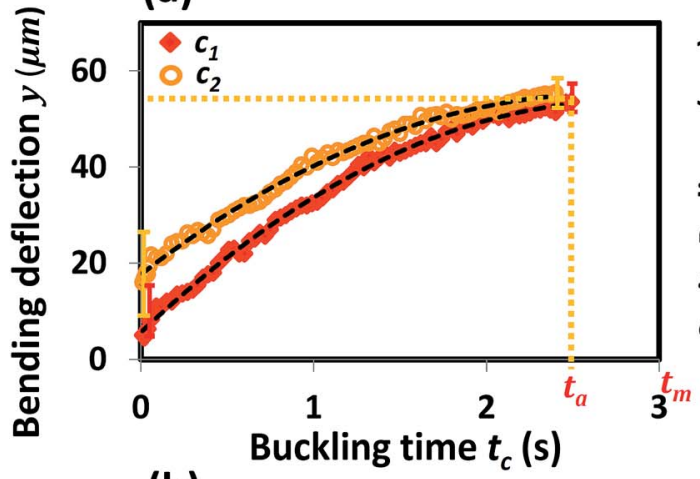

(b)

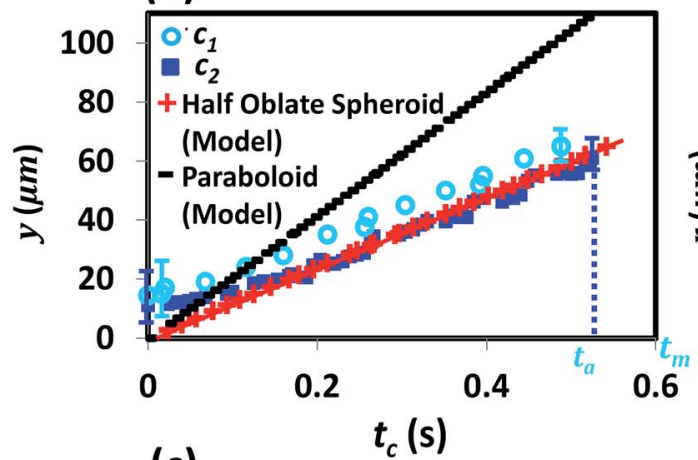

(d)

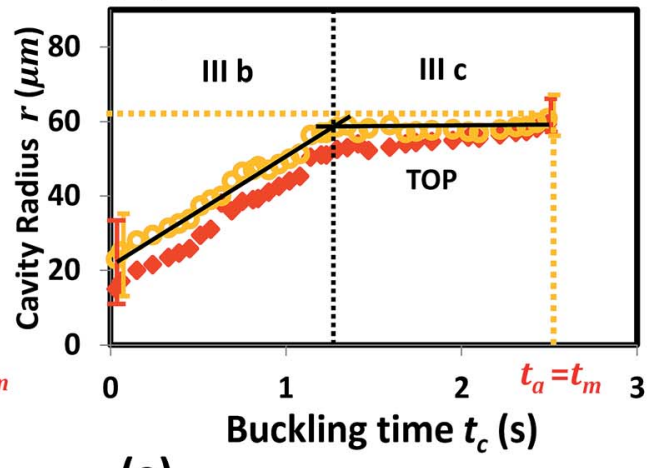

(e)

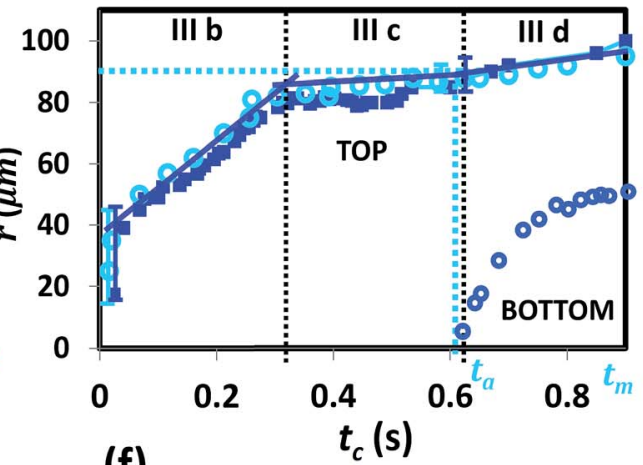

(f)
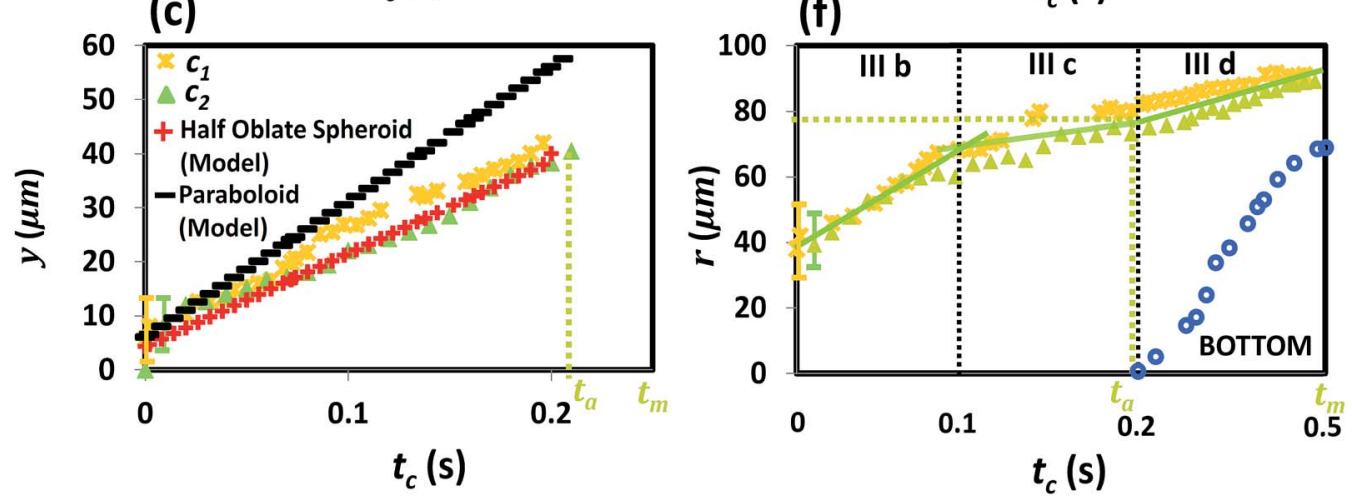

Fig. 8 Temporal variation of the cavity-tip displacement $(y)$ characterizing the cavity growth in the axial direction at (a) $I_{0}=0 \mathrm{MW}^{-2}$ (no heating), (b) $I_{1}=0.104 \mathrm{MW} \mathrm{m}^{-2}$ and (c) $I_{2}=0.208 \mathrm{MW} \mathrm{m}^{-2}$. Three-part, sequential growth of the cavity in the radial direction at the rim (Cavity TOP) and at the equatorial plane (cavity BOTTOM) following cavity growth arrest $\left(t_{\mathrm{c}}=t_{\mathrm{a}}\right.$ ) along the vertical axis, at $(\mathrm{d}) I_{0}=0 \mathrm{MW} \mathrm{m}^{-2}(\mathrm{no}$ heating), (e) $I_{1}=0.104 \mathrm{MW} \mathrm{m}^{-2}$ and (f) $I_{2}=0.208 \mathrm{MW} \mathrm{m}^{-2}$.

or by dynamically stored shell elastic energy can be neglected. The buckling front kinetics are rather governed by the energy and mass transfer effected by solvent vaporization. Since the droplet deformation rate is a direct function of the rate of increase of the shell's elastic deformation energy, these first order estimates show that with an increase in the heating rate the buckling front motion (or cavity spread rate) is accelerated. Based on these energy considerations, the volumetric cavity growth is predicted from the final energy balance as:

$$
2 \frac{\mathrm{d} V_{\mathrm{c}}}{\mathrm{d} t}=\frac{I \pi R_{\mathrm{B}}^{* 2}}{\left(-\rho_{\mathrm{d}} c_{\mathrm{v}} T_{\text {avg }}+\rho_{\mathrm{w}} h_{\mathrm{g}}\right)}
$$

where, the R.H.S. is constant and the L.H.S. is given by $2 \frac{\mathrm{d} V_{\mathrm{c}}}{\mathrm{d} t}=-\frac{\mathrm{d} V_{\mathrm{d}}}{\mathrm{d} t}=$ Rate of volumetric droplet shrinkage (from eqn (3)). Therefore, $-\frac{\mathrm{d} V_{\mathrm{d}}}{\mathrm{d} t}=$ Constant. Since the droplet volume at any instant during the buckling process is $V_{\mathrm{d}}=(\pi / 6)$ $D^{3}$ (where $D$ is the volume equivalent droplet diameter) and given the initial condition that $D=D_{\mathrm{B}}^{*}$ (i.e. the droplet diameter at buckling onset) at time $t_{\mathrm{c}}=0$, then eqn (8) can be written as:

$$
D^{3}(t)=D_{\mathrm{B}}^{* 3}-C t_{\mathrm{c}}
$$

Eqn (8) and (9) have the following implications: first, during the cavity growth phase, droplet surface regression deviates from the linear $D^{2}$-law as the volume-equivalent droplet diameter $D(t)$ exhibits a $t_{\mathrm{c}}^{1 / 3}$ dependence. Second, irrespective of the model assumed for the cavity shape, the volumetric droplet 
deformation rate $-\frac{\mathrm{d} V_{\mathrm{d}}}{\mathrm{d} t}$ is constant. This is only a function of the pre-buckling aspect ratio, the heating rate and the thermophysical properties.

With transitional squeezing of the droplet (due to compressive acoustic radiation pressure at the poles) until a constant aspect ratio is achieved (at the end of the primary deformation phase), the cavity growth is subsequently subjected to a geometric constraint: $y=\frac{h}{2}$ (the maximum bending deflection) at $t=t_{\mathrm{m}}$. This constraint implicitly considers the assistive role played by the acoustic field in controlling the droplet aspect ratio at buckling onset $\left(A R_{\mathrm{B}}\right)$ and hence the maximum cavity growth possible. The buckling front motion characterized by the displacement of the cavity tip is captured by eqn (10) below and is illustrated in Fig. 8

$$
\dot{y}=\frac{0.5\left(I \pi R_{\mathrm{B}}^{* 2}\right)}{k r^{* 2}\left(-\rho_{\mathrm{d}} c_{\mathrm{v}} T_{\text {avg }}+\rho_{\mathrm{w}} h_{\mathrm{g}}\right)}
$$

Eqn (10) indicates that: (1) the bending deflection $(y)$ increases linearly with time (i.e. constant tip velocity) as the buckling fronts move under a dynamic imbalance between the capillary force and the resilient elastic shell deformation (while the solvent percolates and gasifies), and (2) at high vaporization mass flux $\left(I_{1}\right.$ and $\left.I_{2}\right)$ the deformed surface preferentially adopts a half-oblate spheroid geometry compared to a paraboloid. These features are illustrated in Fig. $8 \mathrm{~b}$ and $\mathrm{c}$ and indicate that assuming a paraboloid geometry, the cavity tip growth is significantly over predicted (note that the spherical cap geometry has not been considered to model the cavity since its volume lies mid-way between the assumed curves). In contrast, the experimental data at non lasing conditions shows that the cavity tip growth is rather quadratic in time (Fig. 8a).

\section{Conclusion}

Drying nanoparticle laden droplets suspended in a containerless environment (acoustically levitated) undergo a morphological transition from an initial sphere to a final ring-shaped precipitate structure. This transition is induced by the capillary pressure initiated buckling of a porous elastic crust, which forms through the consolidation of the NPs (as the droplet vaporizes). Based on a global energy balance of the droplet (involving an order of magnitude comparison of the different energy terms), we conclude that during the buckling process droplet gasification deviates from the classical $D^{2}$-law. Particularly, the droplet deformation or the volumetric growth of surface cavities is linear in time and hence exhibits linear variation of $D^{3} v s$. time. Besides, by increasing the input laser flux, both the shell buckling rate (the cavity front motion) and the degree of droplet deformation can be enhanced thereby leading to distinct microstructures. Such controlled manipulation of the buckling front kinetics can be exploited in manufacturing custom-tailored functional microstructures that are crucial to applications ranging from surface patterning to pharmaceutics.

\section{Acknowledgements}

The authors would like to thank Binita Pathak (Research Scholar at Indian Institute of Science) for her help in the experiments, Pooja Sharma (Research Assistant at Indian Institute of Science) for acquiring the SEM images and Dr Debasis Sen (Bhabha Atomic Research Centre, BARC, India) for useful discussions during the course of this work.

\section{References}

1 N. Tsapis, D. Bennett, B. Jackson, D. A. Weitz and D. A. Edwards, Proc. Natl. Acad. Sci. U. S. A., 2002, 72, 12001.

2 K. Masters, Spray Drying Handbook, Halstead Press, New York, 4th edn, 1985.

3 L. Pauchard and Y. Couder, Europhys. Lett., 2004, 66(5), 667. 4 J. Bahadur, D. Sen, S. Mazumder, S. Bhattacharya, H. Frielinghaus and G. Goerigk, Langmuir, 2011, 27(13), 8404-8414.

5 J. Bahadur, D. Sen, S. Mazumder, B. Paul, H. Bhatt and S. G. Singh, Langmuir, 2012, 28(3), 1914-1923.

6 E. Katifori, S. Alben, E. Cerda, D. R. Nelson and J. Dumais, Proc. Natl. Acad. Sci. U. S. A., 2010, 107, 7635-7639.

7 J. Paulose and D. R. Nelson, Buckling pathways in spherical shells with soft spots, Soft Matter, 2013, 9(34), 8227-8245.

8 S. S. Datta, S. H. Kim, J. Paulose, A. Abbaspourrad, D. R. Nelson and D. A. Weitz, Phys. Rev. Lett., 2012, 109(13), 134302.

9 J. Paulose, G. Vliegenthart, G. Gompper and D. R. Nelson, Proc. Natl. Acad. Sci. U. S. A., 2012, 109, 19551.

10 D. Bushnell, AIAA J., 1981, 19(9), 1183-1226.

11 L. Pauchard and C. Allain, Phys. Rev. E: Stat. Phys., Plasmas, Fluids, Relat. Interdiscip. Top., 2003, 68(5), 052801.

12 L. Pauchard and C. Allain, Euro. Phys. Lett., 2003, 62(6), 897.

13 Y. Gorand, L. Pauchard, G. Calligari, J. P. Hulin and C. Allain, Langmuir, 2004, 20(12), 5138-5140.

14 D. Sen, J. S. Melo, J. Bahadur, S. Mazumder, S. Bhattacharya, G. Ghosh and S. F. D'Souza, Eur. Phys. J. E: Soft Matter Biol. Phys., 2010, 31(4), 393-402.

15 A. Sanyal, S. Basu, S. Chowdhuri, P. Kabi and S. Chaudhuri, Appl. Phys. Lett., 2014, 104(16), 163108.

16 D. Sen, J. S. Melo, J. Bahadur, S. Mazumder, S. Bhattacharya, S. F. D'Souza and R. Loidl, Soft Matter, 2011, 7(11), 54235429.

17 S. Sacanna, W. T. M. Irvine, P. M. Chaikin and D. J. Pine, Nature, 2010, 464(7288), 575-578.

18 J. Paulose, G. A. Vliegenthart, G. Gompper and D. R. Nelson, Proc. Natl. Acad. Sci. U. S. A., 2012, 109(48), 19551-19556.

19 A. Fery and R. Weinkamer, Polymer, 2007, 48(25), 7221-7235. 20 E. Tijerino, S. Basu and R. Kumar, J. Appl. Phys., 2013, 113(3), 034307.

21 S. Basu, E. Tijerino and R. Kumar, Appl. Phys. Lett., 2013, $102(14), 141602$.

22 A. L. Yarin, G. Brenn, O. Kastner and C. Tropea, Phys. Fluids, 2002, 14(7), 2289-2298.

23 O. Kastner, G. Brenn, D. Rensink and C. Tropea, Chem. Eng. Technol., 2001, 24(4), 335-339. 
24 B. Park and R. L. Armstrong, Appl. Opt., 1989, 28, 3671-3680. 25 A. L. Yarin, M. Pfaffenlehner and C. Tropea, J. Fluid Mech., 1998, 356, 65-91.

26 A. L. Yarin, G. Brenn, O. Kastner, D. Rensink and C. Tropea, J. Fluid Mech, 1999, 399, 151-204.

27 W. J. Xie and B. Wei, Appl. Phys. Lett., 2007, 90(20), 204104.

28 C. R. Wilke and P. Chang, AIChE J., 1955, 1(2), 264-270.

29 A. Saha, S. Basu, C. Suryanarayana and R. Kumar, Int. J. Heat Mass Trans., 2010, 53(25), 5663-5674.

30 A. Saha, S. Basu and R. Kumar, J. Fluid Mech., 2012, 692, 207219.

31 A. Y. Rednikov, H. Zhao, S. S. Sadhal and E. H. Trinh, Q. J. Mech. Appl. Math., 2006, 59(3), 377-397.
32 V. Y Rudyak, A. A. Belkin and V. V. Egorov, Tech. Phys., 2009, 54(8), 1102-1109.

33 N. Tsapis, E. R. Dufresne, S. S. Sinha, C. S. Riera, J. W. Hutchinson, L. Mahadevan and D. A. Weitz, Phys. Rev. Lett., 2005, 94(1), 018302.

34 L. Landau and E. Lifshitz, Theory of Elasticity, ButterworthHeinemann, 3rd edn, 1986.

35 E. G. Lierke, Acta Acust. Acust., 2002, 88(2), 206-217.

36 L. Pauchard, M. Mermet-Guyennet and F. GiorgiuttiDauphiné, Chem. Eng. Process., 2011, 50(5), 483-485.

37 F. Boulogne, F. Giorgiutti-Dauphiné and L. Pauchard, Soft Matter, 2013, 9(3), 750-757. 